\title{
Quantum Entanglement and Teleportation of Quantum-Dot States in Microcavities*
}

\author{
A. Miranowicz ${ }^{\dagger}$ \\ SORST-JST, Honmachi, Kawaguchi, Saitama 331-0012, Japan, \\ Graduate School of Engineering Science, Osaka University, Toyonaka, Osaka 560-8531, Japan, and \\ Institute of Physics, Adam Mickiewicz University, 61-614 Poznań, Poland \\ Ş. K. Özdemir \\ SORST-JST, Honmachi, Kawaguchi, Saitama 331-0012, Japan, \\ CREST-JST, Honmachi, Kawaguchi, Saitama 331-0012, Japan, and \\ Graduate School of Engineering Science, Osaka University, Toyonaka, Osaka 560-8531, Japan \\ Yu-xi Liu \\ Frontier Research System, Institute of Physical and Chemical Research (RIKEN), Wako-shi 351-0198, Japan \\ G. Chimczak \\ Institute of Physics, Adam Mickiewicz University, 61-614 Poznań, Poland \\ M. Koashi and N. Imoto \\ SORST-JST, Honmachi, Kawaguchi, Saitama 331-0012, Japan, \\ CREST-JST, Honmachi, Kawaguchi, Saitama 331-0012, Japan, and \\ Graduate School of Engineering Science, Osaka University, Toyonaka, Osaka 560-8531, Japan \\ (Received 29 November 2006; Accepted 16 January 2007; Published 9 February 2007)
}

\begin{abstract}
Generation and control of quantum entanglement are studied in an equivalent-neighbor system of spatiallyseparated semiconductor quantum dots coupled by a single-mode cavity field. Generation of genuinely multipartite entanglement of qubit states realized by conduction-band electron-spin states in quantum dots is discussed. A protocol for quantum teleportation of electron-spin states via cavity decay is briefly described.

[DOI: $10.1380 /$ ejssnt.2007.51]
\end{abstract}

Keywords: quantum entanglement; quantum teleportation; quantum dot; qubit

\section{INTRODUCTION}

Among various proposals of scalable quantum computers [1], there has been an increasing interest in quantuminformation processing (QIP) with quantum dots (QDs) [2] since the seminal work of Loss and DiVincenzo [3]. Recently, the interest has further been stimulated by experiments demonstrating the viability of the coherent manipulation of charge states in a single QD [4] and a double QD [5].

Quantum bits (qubits) can be implemented in nanostructures in various ways including electron-spin states (as, e.g., discussed in the next Section), excitonic states [3] or nuclear-spin states [6-8]. The main advantage of the spin qubits is the decoherence times that are a few orders of magnitude longer than the other relevant time scales. However, in a practical implementation of the QD-based quantum computer, one should also be able to (i) quickly induce and control the long-distance couplings between selectively chosen QDs and to (ii) scale such computer to hundreds of qubits, which seemingly require fabrication of high-quality, regularly spaced, uniform semiconductor QDs. A scheme of Imamoğlu, et al. [9] offers a possible

* This paper was presented at Handai Nanoscience and Nanotechnology International Symposium (Handai Nano 2006), Osaka University, Japan, 20-22 November, 2006.

†Corresponding author: miran@amu.edu.pl way to overcome the above-mentioned problems by placing the QDs in a microcavity and illuminating them by laser beams. The long-distance QD interactions are mediated by a single-mode cavity field, and their control is realized by addressing selectively the chosen QDs by laser beams. The possible irregularity of QD structures can be overcome by choosing the proper frequencies and intensities of the laser fields. It is worth noting that a crucial condition for a realization of QIP in such models is a strong coupling of a single QD to a single mode of microcavity (or nanocavity) of a high quality factor (high-Q). Quite recently such random couplings [10] or even deterministic couplings [11] have been observed experimentally.

Quantum entanglement is a key resource for QIP [1]. Here, we extend our former results on bipartite entanglement generation in QDs [12-15] by analysing also generation of multipartite entanglement in the QD systems. We also suggest a protocol for teleportation of QD spin states between distant cavities via their decay. It is a generalization of the scheme for teleportation of atomic-qubit states via cavity decay [16-19].

It is worth stressing that the present analysis is focused on entanglement between electron spins of typical QDs, i.e., of the size smaller than the Bohr radius. In Refs. [13-15, 20], we studied quantum entanglement of excitons in systems of QDs of the size larger than the Bohr radius of an exciton in bulk semiconductor but smaller than the relevant optical wavelengths. In particular, we described realizations of the entangled webs of QD exci- 
tons with symmetric sharing of entanglement, where each $\mathrm{QD}$ is equally entangled to all others. The decoherence of the generated maximally entangled states was studied in greater detail. In particular, we predicted decoherence times as a function of the size of the GaAs and CdS large QDs [20].

\section{A MODEL OF QUANTUM DOTS IN A MICROCAVITY}

We are interested in quantum-information properties of electron-spin states of semiconductor QDs within the model of Imamoğlu, et al. [9], which can be described as follows: The QDs are placed on a microdisk, put into a microcavity tuned to frequency $\omega_{\text {cav }}$, and illuminated selectively by laser fields of frequencies $\omega_{n}^{L}$, where $n$ labels the QDs. Each of $N$ QDs with a single electron in the conduction band is modeled by a three-level atom as shown in Fig. 1. The total Hamiltonian for the three-level QDs interacting with quantized fields reads as follows [12]:

$$
\begin{aligned}
\hat{H} & =\hat{H}_{\mathrm{QD}}+\hat{H}_{\text {fields }}+\hat{H}_{\mathrm{int}}, \\
\hat{H}_{\mathrm{QD}} & =\sum_{n=1}^{N}\left[\mathcal{E}_{n}^{\downarrow} \hat{\sigma}_{n}^{\downarrow \downarrow}+\mathcal{E}_{n}^{\uparrow} \hat{\sigma}_{n}^{\uparrow \uparrow}+\mathcal{E}_{n}^{v} \hat{\sigma}_{n}^{v v}\right], \\
\hat{H}_{\text {fields }} & =\hbar \omega_{\mathrm{cav}} \hat{a}_{\mathrm{cav}}^{\dagger} \hat{a}_{\mathrm{cav}}+\sum_{n=1}^{N} \hbar \omega_{n}^{L}\left(\hat{a}_{n}^{L}\right)^{\dagger} \hat{a}_{n}^{L}, \\
\hat{H}_{\mathrm{int}} & =\sum_{n=1}^{N}\left[\hbar g_{n}^{v \downarrow} \hat{a}_{n}^{L} \hat{\sigma}_{n}^{\downarrow v}+\hbar g_{n}^{v \uparrow} \hat{a}_{\mathrm{cav}} \hat{\sigma}_{n}^{\uparrow v}+\text { h.c. }\right],
\end{aligned}
$$

where $\hat{H}_{\mathrm{QD}}$ and $\hat{H}_{\text {fields }}$ are the free Hamiltonians of the QDs and the fields, respectively; $\hat{H}_{\text {int }}$ is the interaction Hamiltonian; $\hat{a}_{\text {cav }}$ and $\hat{a}_{\text {cav }}^{\dagger}$ are the annihilation and creation operators of the cavity mode, respectively; $\hat{a}_{n}^{L}$ and $\left(\hat{a}_{n}^{L}\right)^{\dagger}$ are the corresponding operators for the laser modes; $\hat{\sigma}_{n}^{x y}=|x\rangle_{n n}\langle y|$ is the $n$th QD operator; $\mathcal{E}_{n}^{(x)}$ is the energy of level $|x\rangle_{n}(x=\downarrow, \uparrow, v)$; the $n$th QD levels $|\downarrow\rangle_{n}$ and $|v\rangle_{n}$ are coupled by dipole interactions with a strength of $g_{n}^{v \downarrow}$; analogously, $g_{n}^{v \uparrow}$ is the coupling strength between levels $|\uparrow\rangle_{n}$ and $|v\rangle_{n}$. There is no direct coupling between levels $|\downarrow\rangle_{n}$ and $|\uparrow\rangle_{m}$ in either the same $(n=m)$ or different QDs $(n \neq m)$. The Hamiltonian (1) simply generalizes, to $N$ QDs and $N+1$ fields, the standard quantum-optical models of a three-level system interacting with two radiation modes (see e.g. [21]).

By applying an adiabatic elimination method, Imamoğlu, et al. [9] derived the effective interaction Hamiltonian

$$
\hat{H}_{\mathrm{eff}}=\frac{\hbar}{2} \sum_{n \neq m} \kappa_{n m}(t)\left(\hat{\sigma}_{n}^{+} \hat{\sigma}_{m}^{-} e^{i\left(\Delta_{n}-\Delta_{m}\right) t}+\text { h.c. }\right)
$$

describing the evolution of the conduction-band spins of $N$ QDs coupled by a microcavity field. This Hamiltonian is given in terms of the Pauli spin creation $\hat{\sigma}_{n}^{+}$and annihilation $\hat{\sigma}_{n}^{-}$operators acting on the conduction-band spin states of the $n$th QD. The effective strength of twoQD coupling between the spins of the $n$th and $m$ th QDs is given by $\kappa_{n m}(t)=g_{n}(t) g_{m}(t) / \Delta_{n}$, where the effective single-QD coupling of the $n$th spin to the cavity field is

\section{conduction band}

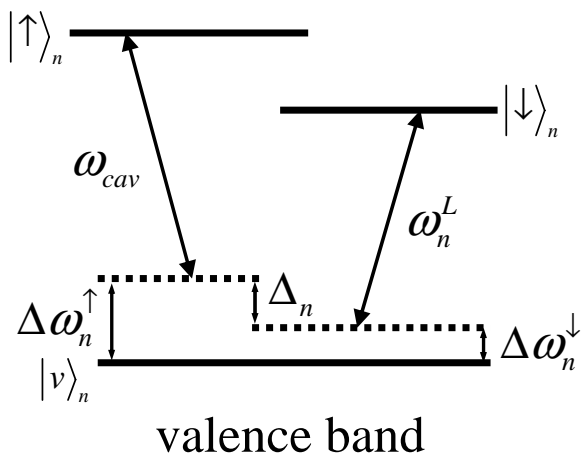

FIG. 1: Effective energy levels of the $n$th QD. Key: $|v\rangle_{n}$ - the effective valence-band state of energy $\mathcal{E}_{n}^{v} ;|\uparrow\rangle_{n}\left(|\downarrow\rangle_{n}\right)$ - spin up (spin down) state of the conduction-band electron of energy $\mathcal{E}_{n}^{\uparrow}\left(\mathcal{E}_{n}^{\downarrow}\right)$; detunings are defined by $\Delta \omega_{n}^{\uparrow}=\mathcal{E}_{n}^{\uparrow}-\mathcal{E}_{n}^{v}-\omega_{\text {cav }}$, $\Delta \omega_{n}^{\downarrow}=\mathcal{E}_{n}^{\downarrow}-\mathcal{E}_{n}^{v}-\omega_{n}^{L}$ and $\Delta_{n}=\Delta \omega_{n}^{\uparrow}-\Delta \omega_{n}^{\downarrow}$.

$g_{n}(t)=g_{n}^{v \downarrow} g_{n}^{v \uparrow}\left|E_{n}^{L}(t)\right| / \Delta \omega_{n}$ with $\Delta \omega_{n}$ being the harmonic mean of $\Delta \omega_{n}^{\uparrow}$ and $\Delta \omega_{n}^{\downarrow}$. For simplicity, the laser fields are assumed to be strong and treated classically as described by the complex amplitudes $E_{n}^{L}(t)$. The Hamiltonian (2) was derived by applying adiabatic eliminations of the valence-band states $|v\rangle_{n}$ and cavity mode $\hat{a}_{\text {cav }}$, which are valid under the assumptions of negligible coupling strength, cavity decay rate, and thermal fluctuations in comparison to $\hbar \Delta_{n}, \hbar \Delta \omega_{n}^{\downarrow}, \hbar \Delta \omega_{n}^{\uparrow}$ and the energy difference $\mathcal{E}_{n}^{\uparrow}-\mathcal{E}_{n}^{\downarrow}$ (see Fig. 1). Moreover, the valence-band levels $|v\rangle_{n}$ were assumed to be far off resonance. Although the Hamiltonian (2) describes apparently direct spin-spin interactions, the real physical picture is different as the quantum-QD spins are coupled only indirectly via the cavity field as described by Hamiltonian (1).

As discussed in Ref. [12], Eq. (2) can be reduced to the effective equivalent-neighbor $N$-QD Hamiltonian

$$
\hat{H}_{\mathrm{eff}}=\frac{\hbar \kappa}{2} \sum_{n \neq m}\left(\hat{\sigma}_{n}^{+} \hat{\sigma}_{m}^{-}+\hat{\sigma}_{n}^{-} \hat{\sigma}_{m}^{+}\right)
$$

even for nonidentical QDs, which can be achieved by adjusting the laser-field frequencies $\omega_{n}^{L}$ to get the same detuning $\Delta_{n}=$ const, and by choosing the proper laser intensities $\left|E_{n}^{L}\right|^{2}$ to obtain the effective coupling constants of $g_{n}(t)=g$ or, equivalently, $\kappa_{n m}(t) \equiv \kappa$ independent of subscripts $n$ and $m$. Thus, in a special case, the model of Imamoğlu, et al. offers a physical realization of the equivalent-neighbor-QD interactions, where each QD interacts with all others with the same strength regardless of their positions or differences in their energetic levels. Note that a system of equivalent-neighbor interactions of up to only four particles can be realized by placing particles in a symmetric geometric configuration. But in the discussed equivalent-neighbor model, the number of QDs can practically be scaled up to $N \sim 100$.

In order to write compactly a solution of the model, it is convenient to introduce the (unnormalized) totally symmetric state

$$
\left|\Phi_{m, n}\right\rangle=\left\{|\uparrow\rangle^{\otimes m}|\downarrow\rangle^{\otimes n}\right\}
$$

as a sum of all $(n+m)$-QD states with $m$ spins up and $n$ spins down. In particular, state (4) for $m=1, n=$ 
1 corresponds to a Bell state (one of the triplet states), $|B\rangle=\frac{1}{\sqrt{2}}\left|\Phi_{1,1}\right\rangle=\frac{1}{\sqrt{2}}(|\uparrow \downarrow\rangle+|\downarrow \uparrow\rangle)$, while for $m=1, n=2$ (and analogously for $m=2, n=1$ ) corresponds to the $W$ state, i.e.:

$$
|W\rangle=\frac{\left|\Phi_{1,2}\right\rangle}{\sqrt{3}}=\frac{1}{\sqrt{3}}(|\uparrow \downarrow \downarrow\rangle+|\downarrow \uparrow \downarrow\rangle+|\downarrow \downarrow \uparrow\rangle) .
$$

Let us assume that the initial state describing a system of $M(M=0, \ldots, N)$ QDs with spin up (of a single conduction-band electron) and $(N-M)$ QDs with spin down is $\left|\psi^{N M}(0)\right\rangle=|\uparrow\rangle^{\otimes M}|\downarrow\rangle^{\otimes(N-M)}=$ $\left|\Phi_{M, 0}\right\rangle\left|\Phi_{0, N-M}\right\rangle$. Then the solution of the Schrödinger equation of motion for Hamiltonian (3) is given by [12]:

$$
\left|\psi^{N M}(t)\right\rangle=\sum_{m=0}^{M^{\prime}} \gamma_{m}^{N M}(t)\left|\Phi_{M-m, m}\right\rangle\left|\Phi_{m, N-M-m}\right\rangle .
$$

The time-dependent superposition coefficients are given by

$$
\begin{aligned}
\gamma_{m}^{N M}(t) & =\sum_{n=0}^{M^{\prime}} b_{n m}^{N M} e^{i[n(N+1-n)-M(N-M)] \kappa t} \\
b_{n m}^{N M} & =\sum_{k=0}^{m}(-1)^{k} \frac{C_{k}^{m}}{C_{M-k}^{N-2 k}}\left(C_{n-k}^{N+1-2 k}-2 C_{n-k-1}^{N-2 k}\right)(8)
\end{aligned}
$$

where $M^{\prime}=\min (M, N-M)$ and $C_{y}^{x}$ are binomial coefficients. For $N$-QD systems initially in $\left|\psi^{N 1}(0)\right\rangle=$ $|\uparrow\rangle|\downarrow\rangle^{\otimes(N-1)}$, solution (6) simplifies to:

$$
\left|\psi^{N 1}(t)\right\rangle=\gamma_{0}^{N 1}(t)|\uparrow\rangle|\downarrow\rangle^{\otimes(N-1)}+\gamma_{1}^{N 1}(t)|\downarrow\rangle\left|\Phi_{1, N-2}\right\rangle .
$$

We will apply and analyze quantum properties of these solutions in the next section.

\section{ENTANGLEMENT OF ELECTRON-SPIN STATES}

Here, we analyze generation of quantum entanglement of electron-spin states of semiconductor QDs within the discussed equivalent-neighbor model. We will describe various kinds of entanglement as depicted schematically in Fig. 2 including pure- and mixed-state bipartite entanglement, as well as pure-state genuine multipartite entanglement.

In the analysis of the pure-state bipartite entanglement, it is useful to decompose the solution (6) as follows

$$
\left|\psi^{N M}(t)\right\rangle=\sum_{m=0}^{M^{\prime}} \sqrt{P_{m}^{N M}(t)}\left|\phi_{m}(t)\right\rangle_{A}\left|\varphi_{m}(t)\right\rangle_{B}
$$

in terms of the orthonormal-basis states $\left|\phi_{m}(t)\right\rangle_{A}$ and $\left|\varphi_{m}(t)\right\rangle_{B}$ for subsystems $A$ and $B$, respectively, and the Schmidt coefficients given by

$$
P_{m}^{N M}(t)=C_{m}^{M} C_{m}^{N-M}\left|\gamma_{m}^{N M}(t)\right|^{2} .
$$

Eq. (11) directly enables us calculation of the von Neumann entropy

$$
E^{N M}(t)=-\sum_{m=0}^{M^{\prime}} P_{m}^{N M}(t) \log _{2} P_{m}^{N M}(t),
$$

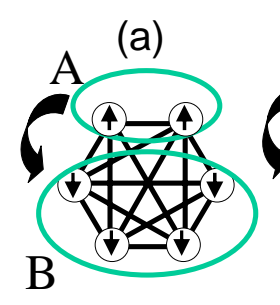

(d)

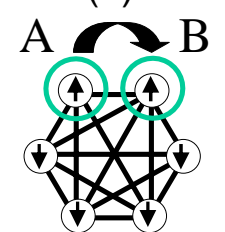

(b)

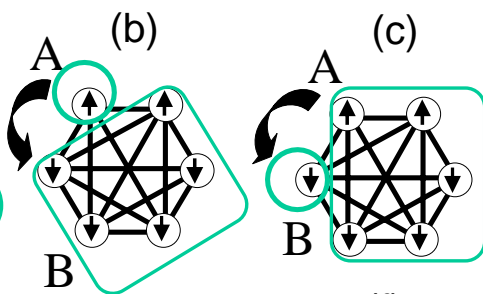

(e)

(f)

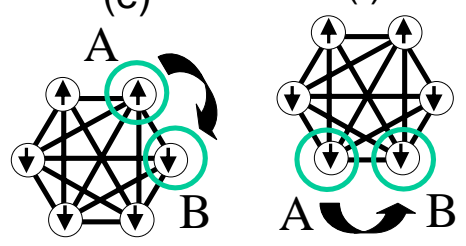

FIG. 2: Schematic representation of various bipartite entanglements between subsystems $A$ and $B$ in a system of $N$ equivalent-neighbor QDs: (a) pure-state entanglement between $M$ QDs initially with spin up and the remaining $(N-M)$ QDs with spin down as described by the von Neumann entropy $E^{N M}(t) ;(\mathrm{b}, \mathrm{c})$ pure-state entanglement between a given QD (initially either with spin up or down) and the rest of the system being described by tangle $\tau_{\uparrow \tilde{\uparrow}}^{N M}(t)$ or $\tau_{\downarrow \tilde{\downarrow}}^{N M}(t)$ respectively; $(\mathrm{d}, \mathrm{e}, \mathrm{f})$ three types of mixed-state pairwise entanglement described by concurrences $C_{\uparrow \uparrow}^{N M}(t), C_{\uparrow \downarrow}^{N M}(t)$, and $C_{\downarrow \downarrow}^{N M}(t)$, respectively. Analysis of bipartite entanglement shown in figures (b)-(f) enables extraction of information about intrinsic multipartite entanglement.

as the Shannon entropy of the Schmidt coefficients. This formula determines bipartite entanglement between the QDs initially with spin up (subsystem $A$ ) and the remaining QDs (subsystem $B$ ), as shown in Fig. 2(a). It is easy to see that it holds the following symmetry of the Schmidt coefficients $P_{m}^{N, M}(t)=P_{m}^{N, N-M}(t)$, which implies that the entanglements for systems with $M$ and $(N-M)$ QDs initially with spin up are the same for any evolution times.

In Ref. [12], we addressed the question of generation of maximum entanglement between two subsystems $A$ and $B$ consisting of $M$ and $(N-M)$ QDs, respectively. The simplest nontrivial evolution of our system occurs for two QDs with one of them initially with spin up given by

$$
\begin{aligned}
\left|\psi^{21}(t)\right\rangle & =\gamma_{0}^{21}(t)|\uparrow \downarrow\rangle+\gamma_{1}^{21}(t)|\downarrow \uparrow\rangle \\
& =\cos (\kappa t)|\uparrow \downarrow\rangle-i \sin (\kappa t)|\downarrow \uparrow\rangle,
\end{aligned}
$$

as a special case of (9). The state periodically evolves into Bell-like states $\left|\psi^{21}\left(t^{\prime}\right)\right\rangle=\frac{1}{\sqrt{2}}(|\uparrow \downarrow\rangle \pm i|\downarrow \uparrow\rangle)$ at times $\kappa t^{\prime}=$ $(2 n+1) \frac{\pi}{4}(n=0,1, \ldots)$. In general, from the Bennett, et al. theorem [22] follows that the maximally entangled states has the entanglement of $\log _{2}\{\min (M, N-M)+1\}$ ebits. For our systems under interactions described by (3) with a single spin up $(M=1)$, the Schmidt coefficients (11) reduce to $P_{1}^{N 1}(t)=4(N-1) N^{-2} \sin ^{2}\left(\frac{N}{2} \kappa t\right)$ and $P_{0}^{N 1}(t)=1-P_{1}^{N 1}(t)$, which enable a direct calculation of the entanglement $E^{N 1}(t)$ and its maximum values from Eq. (12). One can find [12] that the maxima can be observed at the times $\kappa t^{\prime}= \pm \frac{2}{N} \operatorname{arccsc}\left(\frac{2}{N} \sqrt{2(N-1)}\right)+n T$ and $\kappa t^{\prime \prime}=\frac{\pi}{N}+n T$ with $n=0,1, \cdots$ multiples of the period $T \equiv T^{N 1}=\frac{2 \pi}{N}$. Then it is easy to prove that the maximally entangled state (EPR state) with $E^{N 1}\left(t^{\prime}\right)=1$ ebit, can be achieved at the evolution times $t^{\prime}$ for $N \leq 6$ only. For $N>6$, any real solution for $t^{\prime}$ does not exist, 
and the entanglement reaches its maximum at the evolution times $t^{\prime \prime}$ but $E^{N 1}\left(t^{\prime \prime}\right)=\max _{t} E^{N 1}(t)$ is less than one ebit, thus the EPR state cannot be generated exactly in the systems having more than six QDs, where one of them has initially spin opposite to the spin of the other QDs. A numerical analysis shows that if the number $M$ of initial spins up is $1<M<N-1$ of any number $N$ of QDs then our system will not arrive at the exact EPR states either. A sudden decrease of entanglement is observed on increasing the total number of QDs in a system with a fixed number of QDs with spin up. Nevertheless, very good approximations of the EPR states with almost $\log _{2}([N / 2]+1)$ ebits of entanglement can be achieved periodically if the system has half (or almost half) spins up, $M=[N / 2][12]$.

To analyze a genuine multipartite entanglement, we apply the approach proposed by Coffman, Kundu and Wootters $(\mathrm{CKW})[23]$ via the so-called tangles and monogamy inequality. Let us define the tangle $\tau\left(\hat{\rho}_{n \tilde{n}}\right)$ as the entanglement measure between the $n$th qubit and all the remaining ones (denoted by $\tilde{n}$ ), which corresponds to entanglement schematically depicted in Figs. 2(b,c). The tangle for arbitrary mixed state $\hat{\rho}=\hat{\rho}_{n \tilde{n}}$ of the $2 \times d$ system is defined as the convex roof [24]:

$$
\tau\left(\hat{\rho}_{n \tilde{n}}\right)=\inf _{\left\{p_{i},\left|\psi_{i}\right\rangle\right\}} \sum_{i} p_{i} S\left[\operatorname{tr}_{\tilde{n}}\left(\left|\psi_{i}\right\rangle\left\langle\psi_{i}\right|\right)\right]
$$

of the single-qubit linear entropy

$$
S(\hat{\rho})=2\left[1-\operatorname{tr}\left(\hat{\rho}^{2}\right)\right]=4 \operatorname{det}(\hat{\rho}),
$$

where the infimum is taken over all pure-state decompositions $\left\{p_{i},\left|\psi_{i}\right\rangle\right\}$ of $\hat{\rho}=p_{i}\left|\psi_{i}\right\rangle\left\langle\psi_{i}\right|$. For pure states, as analyzed in our paper, the tangle is simply defined as $\tau\left(\hat{\rho}_{n \tilde{n}}\right)=S\left[\operatorname{tr}_{\tilde{n}}\left(\hat{\rho}_{n \tilde{n}}\right)\right]$.

A pairwise entanglement between any two QDs, as depicted schematically in Figs. 2(d,e,f), can be described by the concurrence, defined by [25]

$$
C(\hat{\rho})=\max \left\{0,2 \max _{i} \lambda_{i}-\sum_{i=1}^{4} \lambda_{i}\right\}
$$

for a reduced two-qubit mixed state $\hat{\rho}$. In definition (16), $\lambda_{i}$ are the square roots of the eigenvalues of the matrix $\hat{\rho}\left(\hat{\sigma}_{y} \otimes \hat{\sigma}_{y}\right) \hat{\rho}^{*}\left(\hat{\sigma}_{y} \otimes \hat{\sigma}_{y}\right)$, where $\hat{\sigma}_{y}$ is the Pauli spin matrix and the asterisk denotes complex conjugation. The concurrence is related to the entanglement of formation, $E_{F}(\hat{\rho})$, defined as the minimum mean entanglement of an ensemble of pure states $\left|\psi_{i}\right\rangle$ that represents $\hat{\rho}[26]: E_{F}(\hat{\rho})=\min _{\left\{p_{i},\left|\psi_{i}\right\rangle\right\}} \sum_{i} p_{i} E\left(\left|\psi_{i}\right\rangle\left\langle\psi_{i}\right|\right)$, where $\hat{\rho}=\sum_{i} p_{i}\left|\psi_{i}\right\rangle\left\langle\psi_{i}\right|$ and $E\left(\left|\psi_{i}\right\rangle\left\langle\psi_{i}\right|\right)$ is the entropy of entanglement of pure state $\left|\psi_{i}\right\rangle$ defined by the von Neumann entropy. As shown by Wootters [25], the entanglement of formation for two qubits in an arbitrary mixed state $\hat{\rho}$ can explicitly be given in terms of the concurrence as follows $E_{F}(\hat{\rho})=H\left(\frac{1}{2}\left[1+\sqrt{1-C(\hat{\rho})^{2}}\right]\right)$, where $H(x)$ is the Shannon binary entropy. Note that $E_{F}(\hat{\rho})$ and $C(\hat{\rho})$ are monotonic functions of one another and both range from 0 (for a separable state) to 1 (for a maximally entangled state).

CKW conjectured that the bipartite entanglement of multipartite qubit states satisfies the following monogamy inequality [23]

$$
\Delta\left(\hat{\rho}_{n}\right)=\tau\left(\hat{\rho}_{n \tilde{n}}\right)-\sum_{m(m \neq n)} C^{2}\left(\hat{\rho}_{n m}\right) \geq 0
$$

as quantified by the squared concurrence $C^{2}\left(\hat{\rho}_{n m}\right)$ and the tangle $\tau\left(\hat{\rho}_{n \tilde{n}}\right)$. The conjecture was first proved by CKW for 3 qubits in pure state [23], but a general proof for arbitrary number of qubits in arbitrary states has been given only recently by Osborne and Verstraete [24]. We use the quantity $\Delta\left(\hat{\rho}_{n}\right)$, which is called the residual tangle, as a criterion for genuine (or intrinsic) multipartite entanglement of qubit states, i.e. for correlations not stored in two-qubit entanglement. In particular, the residual tangle for $N=3$ qubits is referred as the 3-tangle and describes genuine 3-partite (3-way) entanglement. In general, for $N>3$ qubits, $\Delta\left(\hat{\rho}_{n}\right)$ contains information about not solely $N$-way entanglement, but rather all kinds of $3,4, \ldots, N$-partite entanglements.

As the main new result of this paper, we analyze a possibility to generate an intrinsic multipartite entanglement in our model as being described by the CKW inequality. For our equivalent-neighbor system, there are only three different types of evolution of the concurrence depending on the choice of a pair of QDs as described by the reduced density matrices: (i) $\hat{\rho}_{\uparrow \uparrow}^{N M}=\operatorname{tr}_{3,4, \ldots, N}\left(\hat{\rho}^{N M}\right)$ for both QDs initially with spin up corresponding, in particular, to two qubits shown in Fig. 2(d), (ii) $\hat{\rho}_{\uparrow \downarrow}^{N M}=$ $\operatorname{tr}_{1,2, \ldots, M-1, M+2, \ldots, N}\left(\hat{\rho}^{N M}\right)$ for a pair of QDs when initially one of them has spin up and the other has spin down (see Fig. 2(e)), and (iii) $\hat{\rho}_{\downarrow \downarrow}^{N M}=\operatorname{tr}_{1,2, \ldots, N-2}\left(\hat{\rho}^{N M}\right)$ for both QDs initially with spin down (see Fig. 2(f)), where $\hat{\rho}^{N M}=\left|\psi^{N M}\right\rangle\left\langle\psi^{N M}\right|$. By properly grouping terms of Eq. (6) and applying partial trace, we find the following general solutions for the three kinds of the reduced density matrices:

$$
\begin{array}{r}
\hat{\rho}_{\uparrow \downarrow}^{N M}(t)=\alpha_{\uparrow \downarrow 0}(t)|\downarrow \downarrow\rangle\left\langle\downarrow \downarrow\left|+\alpha_{\uparrow \downarrow 1}(t)\right| \uparrow \uparrow\right\rangle\langle\uparrow \uparrow| \\
+\sum_{m=0}^{M^{\prime}-1} C_{m}^{M-1} C_{m}^{N-M-1}\left|\beta_{m}(t)\right\rangle\left\langle\beta_{m}(t)\right|,
\end{array}
$$

where $(j=0,1)$

$$
\begin{aligned}
\alpha_{\uparrow \downarrow j}(t) & =\sum_{m=0}^{M^{\prime}}\left|\gamma_{m}^{N M}(t)\right|^{2} C_{m-1+j}^{M-1} C_{m-j}^{N-M-1}, \\
\left|\beta_{m}(t)\right\rangle & =\gamma_{m}^{N M}(t)|\uparrow \downarrow\rangle+\gamma_{m+1}^{N M}(t)|\downarrow \uparrow\rangle,
\end{aligned}
$$

and for $k=\uparrow \uparrow, \downarrow \downarrow$ :

$$
\begin{aligned}
\hat{\rho}_{k}^{N M}(t) & =\alpha_{k 0}(t)|\downarrow \downarrow\rangle\left\langle\downarrow \downarrow\left|+2 \alpha_{k 1}(t)\right| B\right\rangle\langle B| \\
& +\alpha_{k 2}(t)|\uparrow \uparrow\rangle\langle\uparrow \uparrow|,
\end{aligned}
$$

where $(j=0,1,2)$

$$
\begin{aligned}
& \alpha_{\uparrow \uparrow j}(t)=\sum_{m=0}^{M^{\prime}}\left|\gamma_{m}^{N M}(t)\right|^{2} C_{m}^{N-M} C_{m-2+j}^{M-2}, \\
& \alpha_{\downarrow \downarrow j}(t)=\sum_{m=0}^{M^{\prime}}\left|\gamma_{m}^{N M}(t)\right|^{2} C_{m}^{M} C_{m-j}^{N-M-2} .
\end{aligned}
$$



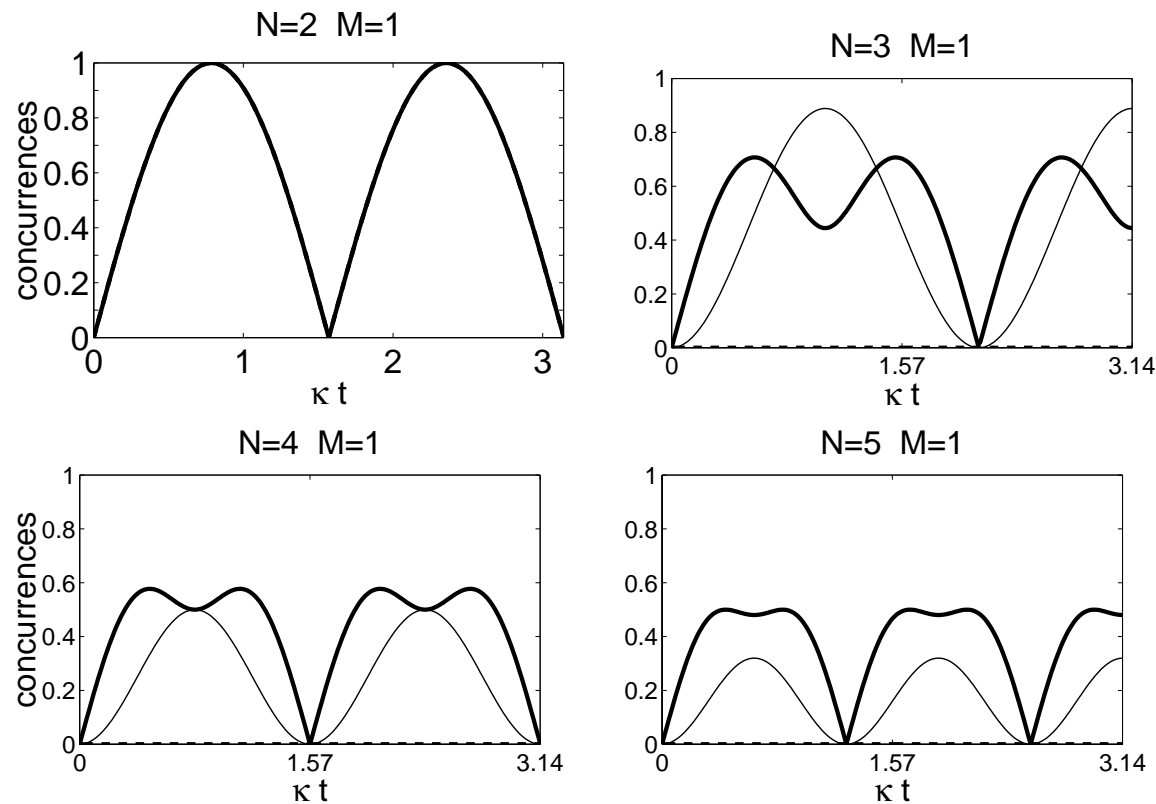

$\mathrm{N}=6 \quad \mathrm{M}=1$
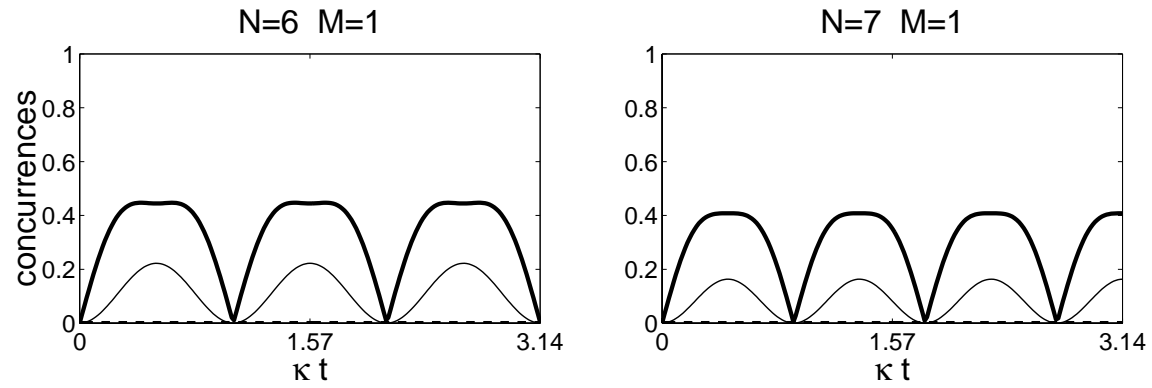

FIG. 3: Evolution of concurrence for systems of $N$ QDs with only a single QD initially with spin up $(M=1)$ : $C_{\uparrow \downarrow}^{N 1}(t)($ thick curves) and $C_{\downarrow \downarrow}^{N 1}(t)$ (thin curves). Note that the residual tangles are vanishing, $\Delta_{\uparrow}^{N 1}(t)=\Delta_{\downarrow}^{N 1}(t)=0$.

For simplicity, we dropped superscripts $N M$ in $\alpha_{k j}$ and $\left|\beta_{m}(t)\right\rangle$. In the following we use the following shorthand notation: $C_{\uparrow \uparrow}^{N M}(t) \equiv C\left(\hat{\rho}_{\uparrow \uparrow}^{N M}(t)\right), \tau_{\uparrow \tilde{\uparrow}}^{N M}(t) \equiv \tau\left(\hat{\rho}_{\uparrow \tilde{\uparrow}}^{N M}(t)\right)$, $\Delta_{\uparrow}^{N M}(t) \equiv \Delta\left(\hat{\rho}_{\uparrow}^{N M}(t)\right)$, etc.

In Figs. 3-5, we present examples of the concurrence evolution according to solutions for the reduced density matrices, given by (18) and (21), assuming $M=1$ and $M=[N / 2]$. Note that $C_{\uparrow \uparrow}^{N 1}(t)=0$ as the system has only single spin up during the whole evolution. Analogously, one can find that $C_{\uparrow \uparrow}^{2 M, M}(t)=C_{\downarrow \downarrow}^{2 M, M}(t)=0$ for any $M$. By analyzing the figures, one can clearly see that the maximum of concurrences decreases but the maximum of residual tangles increases with increasing number $N$ of QDs for $M>1$.

In our equivalent-neighbor model, we find only two kinds of tangles $\tau_{k \tilde{k}}^{N M}$ corresponding to a QD initially with spin up $(k=\uparrow)$ or spin down $(k=\downarrow)$ as given by

$$
\tau_{k \tilde{k}}^{N M}(t)=4 \alpha_{k}(t)\left[1-\alpha_{k}(t)\right]
$$

which corresponds to the reduced density matrices $\rho_{k \tilde{k}}^{N M}(t)=\alpha_{k}(t)|\downarrow\rangle\left\langle\downarrow\left|+\left[1-\alpha_{k}(t)\right]\right| \uparrow\right\rangle\langle\uparrow|$, where coefficients $\alpha_{k}(t)=\alpha_{k 0}(t)+\alpha_{k 1}(t)=1-\alpha_{k 1}(t)-\alpha_{k 2}(t)$ are given in terms of (22). The tangle, given by (23), describes entanglement between the $k$ th $\mathrm{QD}$ and all the remaining QDs (denoted by subscript $\tilde{k}$ ). The residual tangles $\Delta_{k}^{N M} \equiv \Delta\left(\hat{\rho}_{k}^{N M}\right)$ are then given by

$$
\begin{aligned}
\Delta_{\uparrow}^{N M}(t)= & \tau_{\uparrow \tilde{\uparrow}}^{N M}(t)-(M-1)\left[C_{\uparrow \uparrow}^{N M}(t)\right]^{2} \\
& -(N-M)\left[C_{\uparrow \downarrow}^{N M}(t)\right]^{2}, \\
\Delta_{\downarrow}^{N M}(t)= & \tau_{\downarrow \tilde{\downarrow}}^{N M}(t)-(N-M-1)\left[C_{\downarrow \downarrow}^{N M}(t)\right]^{2} \\
& -M\left[C_{\uparrow \downarrow}^{N M}(t)\right]^{2} .
\end{aligned}
$$

Let us analyze a few examples of the general solutions to describe generation of $N$-partite entanglement. The evolution, given by the solution (9), of the initial state $\left|\psi^{31}(0)\right\rangle=|\uparrow \downarrow \downarrow\rangle$ for three QDs with a single spin up can explicitly be given by

$$
\left|\psi^{31}(t)\right\rangle=\gamma_{0}^{31}(t)|\uparrow \downarrow \downarrow\rangle+\sqrt{2} \gamma_{1}^{31}(t)|\downarrow\rangle|B\rangle,
$$

where $\gamma_{0}^{31}(t)=\left(e^{-i 2 \kappa t}+2 e^{i \kappa t}\right) / 3$ and $\gamma_{1}^{31}(t)=\left(e^{-i 2 \kappa t}-\right.$ $\left.e^{i \kappa t}\right) / 3$. The state periodically arrives at times $\kappa t^{\prime}=(9 n+$ 1) $\frac{2 \pi}{9}$ into a $W$ state:

$$
\left|\psi^{31}\left(t^{\prime}\right)\right\rangle=\frac{1}{\sqrt{3}}\left(\mathrm{e}^{i \theta_{0}}|\uparrow \downarrow \downarrow\rangle+\mathrm{e}^{i \theta_{1}}|\downarrow \uparrow \downarrow\rangle+\mathrm{e}^{i \theta_{1}}|\downarrow \downarrow \uparrow\rangle\right)
$$

deviating from the standard $W$ state by the phases $\theta_{0}=$ $\arctan \left(\frac{\sqrt{3} s-c}{\sqrt{3} c+s}\right)$ and $\theta_{1}=\arctan \left(\frac{\sqrt{3} s+c}{\sqrt{3} c-s}\right)-\pi$, where $s=\sin (2 \pi / 9)$ and $c=\cos (2 \pi / 9)$. For the $W$ state one finds from our general solution that the concurrences are the same and equal to $C_{\uparrow \downarrow}^{31}\left(t^{\prime}\right)=C_{\downarrow \downarrow}^{31}\left(t^{\prime}\right)=2 / 3$, and the 

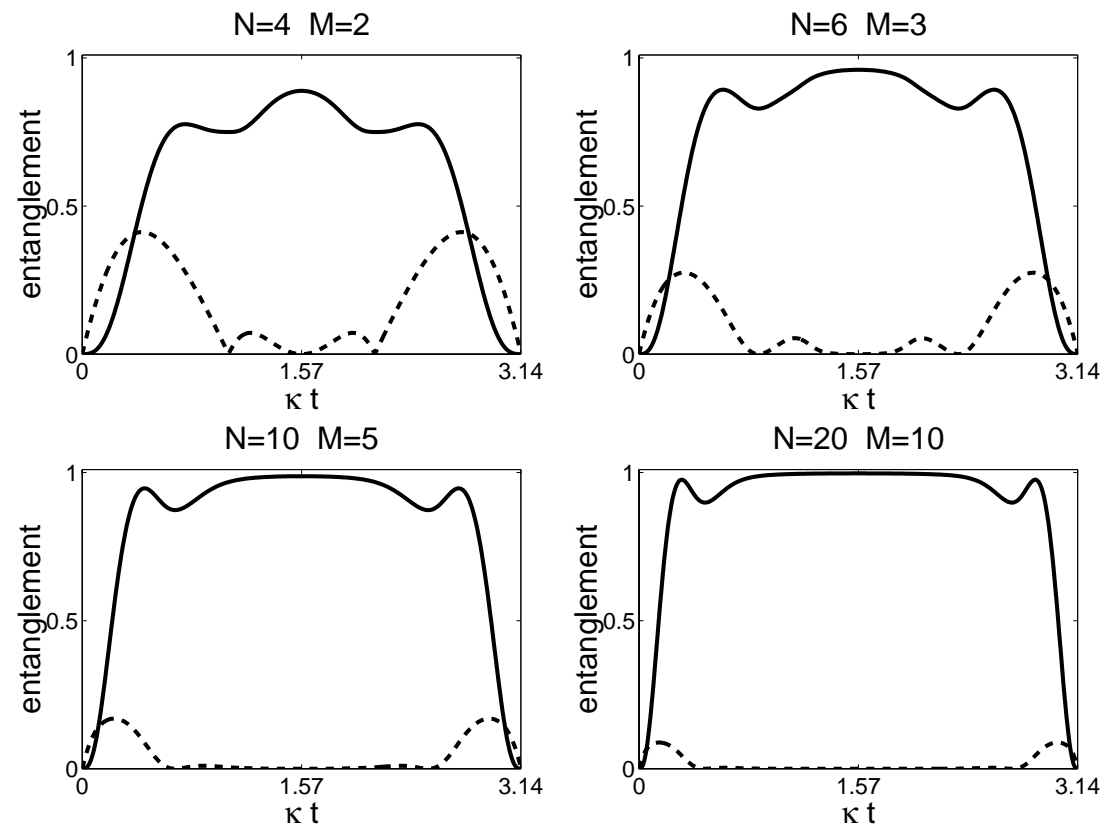

FIG. 4: Evolution of the residual tangles $\Delta_{\uparrow}^{N M}(t)=\Delta_{\downarrow}^{N M}(t)$ (solid curves) and the concurrence $C_{\uparrow \perp}^{N M}(t)$ (broken curves) for systems of $N$ QDs with half of them with spin up. Note that $C_{\downarrow \downarrow}^{N M}(t)=C_{\uparrow \uparrow}^{N M}(t)=0$.
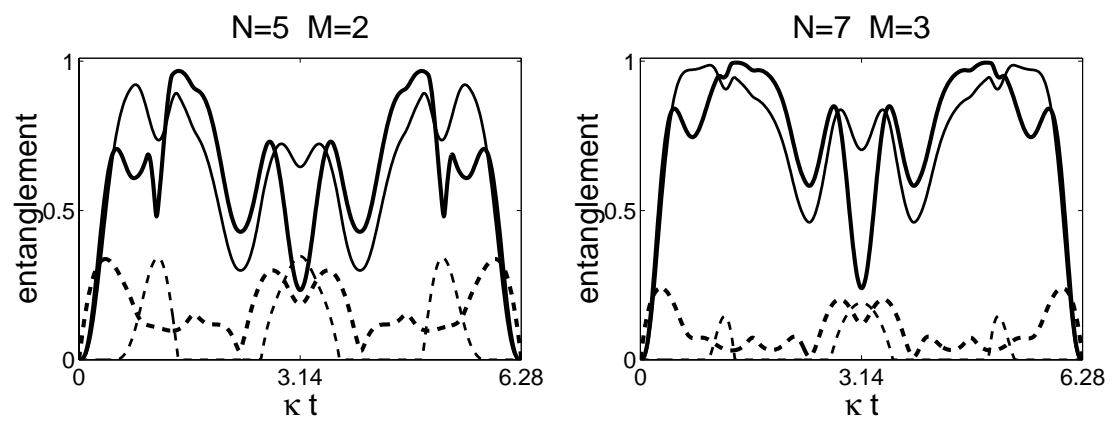

FIG. 5: Evolution of the residual tangles $\Delta_{\uparrow}^{N M}(t)$ (thick solid curves) and $\Delta_{\downarrow}^{N M}(t)$ (thin solid) as well the concurrences $C_{\uparrow \downarrow}^{N M}(t)$ (thick broken curves) and $C_{\downarrow \downarrow}^{N M}(t)$ (thin broken) for systems of $N=2 M+1$ QDs. For clarity we omit curves for $C_{\uparrow \uparrow}^{N M}(t)$.

tangles are $\tau_{\uparrow \uparrow}^{31}\left(t^{\prime}\right)=\tau_{\downarrow \tilde{\downarrow}}^{31}\left(t^{\prime}\right)=8 / 9$, while the residual tangles are vanishing, $\Delta_{\uparrow}^{31}\left(t^{\prime}\right)=\Delta_{\downarrow}^{31}\left(t^{\prime}\right)=0$. Thus, it is clear the state does not exhibit intrinsic 3-particle entanglement in agreement with the CKW result [23]. For other evolution times, it is impossible to get higher value of the mutually equal concurrences (and the tangles) as the $\mathrm{W}$ state reaches the upper bound of $2 / N$ for symmetric sharing of entanglement [27, 28]. Nevertheless one of the tangles or the concurrences can be larger, at a given moment, than those for the $W$ state. On the other hand, the residual tangles are always vanishing. These conclusions can be drawn by analyzing the explicit solutions:

$$
\begin{aligned}
\tau_{\downarrow \tilde{\downarrow}}^{31}(t) & =\left[C_{\uparrow \downarrow}^{31}(t)\right]^{2}+\left[C_{\downarrow \downarrow}^{31}(t)\right]^{2}=\tau^{\prime}[7+2 \cos (3 \kappa t)], \\
\tau_{\uparrow \uparrow}^{31}(t) & =2\left[C_{\uparrow \downarrow}^{31}(t)\right]^{2}=2 \tau^{\prime}[5+4 \cos (3 \kappa t)],
\end{aligned}
$$

where $\tau^{\prime}=(16 / 81) \sin ^{2}(3 \kappa t / 2)$. Thus, one can observe that the maximum of concurrence $\max _{t} C_{\uparrow \downarrow}^{31}(t)=C_{\uparrow \downarrow}^{31}\left(t^{\prime \prime}\right)$ is $8 / 9$ and the maximum of the tangle $\max _{t} \tau_{\uparrow \uparrow}^{31}(t)=$ $\tau_{\uparrow \widetilde{\uparrow}}^{31}\left(t^{\prime \prime}\right)$ reaches $80 / 81$ for the moments $\kappa t^{\prime \prime}= \pm 0.565 \cdots+$ $n 2 \pi / 3(n=0,1, \ldots)$. On the other hand, $\max _{t} C_{\downarrow \downarrow}^{31}(t)=$ $C_{\downarrow \downarrow}^{31}\left(t^{\prime \prime \prime}\right)=8 / 9$ and $\max _{t} \tau_{\downarrow \tilde{\downarrow}}^{31}(t)=\tau_{\downarrow \tilde{\downarrow}}^{31}\left(t^{\prime \prime \prime}\right)=0.9877$ for the moments $\kappa t^{\prime \prime \prime}=(2 n+1) \pi / 3$. It is seen that these values of the entanglement measures are much higher than those for the $W$ state.
Similarly for $N=4$, the state $\left|\psi^{41}(0)\right\rangle$ evolves at times $\kappa t^{\prime}=(2 n-1) \frac{\pi}{4}$ into a four-particle (generalized) $W$ state

$$
\left|\psi^{41}\left(t^{\prime}\right)\right\rangle \sim \frac{1}{2}(|\uparrow \downarrow \downarrow \downarrow\rangle-|\downarrow \uparrow \downarrow \downarrow\rangle-|\downarrow \downarrow \uparrow \downarrow\rangle-|\downarrow \downarrow \downarrow \uparrow\rangle) .
$$

Due to the symmetry of the system, $W$ states are also generated for $\left|\psi^{32}(t)\right\rangle$ and $\left|\psi^{43}(t)\right\rangle$. However, as can be shown semi-analytically and numerically, the states $\left|\psi^{N M}(0)\right\rangle$ for $N=4, M=2$ as well as for $N>4$ with any $M$ do not evolve into the exact $W$ states under the interaction described by Hamiltonian (2). The concurrences $C_{\uparrow \downarrow}^{41}\left(t^{\prime}\right)=C_{\downarrow \downarrow}^{41}\left(t^{\prime}\right)$ reach the value $1 / 2$, which is the upper bound of symmetric sharing of entanglement for four qubits [27, 28]. Additionally, the tangles are found to be $\tau_{\uparrow \uparrow}^{41}\left(t^{\prime}\right)=\tau_{\downarrow \widetilde{l}}^{41}\left(t^{\prime}\right)=3 / 4$. As in the former example, we want to get higher-values of the tangles and concurrences by resigning from the condition that $C_{\uparrow \downarrow}^{41}\left(t^{\prime}\right)=C_{\downarrow \downarrow}^{41}\left(t^{\prime}\right)$. From the general solutions, one gets

$$
\begin{aligned}
& \tau_{\downarrow \tilde{\downarrow}}^{41}(t)=\left[C_{\uparrow \downarrow}^{41}(t)\right]^{2}+2\left[C_{\downarrow \downarrow}^{41}(t)\right]^{2}=\tau^{\prime}[7+\cos (4 \kappa t)] \\
& \tau_{\uparrow \uparrow}^{41}(t)=3\left[C_{\uparrow \downarrow}^{41}(t)\right]^{2}=3 \tau^{\prime}[5+3 \cos (4 \kappa t)]
\end{aligned}
$$

where $\tau^{\prime}=(1 / 8) \sin ^{2}(2 \kappa t)$. Thus, one can find that maximum of concurrence $C_{\uparrow \downarrow}^{41}(t)$ (equal to 0.577 ) and maximum of tangle $\tau_{\uparrow \uparrow}^{41}(t)$ (equal to 1 ) are higher than those 
TABLE I: Maxima of the concurrences $C_{\uparrow \uparrow}^{N M}(t), C_{\uparrow \downarrow}^{N M}(t)$ and $C_{\downarrow \downarrow}^{N M}(t)$, the tangles $\tau_{\downarrow \tilde{\downarrow}}^{N M}(t)$ as well as the residual tangles $\Delta_{\uparrow}^{N M}(t)$ and $\Delta_{\uparrow}^{N M}(t)$ for equivalent-neighbor system of $N$ QDs with $M$ of them initially with spin up. Additionally, $\max _{t} \tau_{\uparrow \uparrow}^{N M}(t)=1.000$ for all presented cases except $\max _{t} \tau_{\uparrow \widetilde{\uparrow}}^{10,2}(t)=0.991$. Note that for other values of $M$, the maxima of the tangles $\tau_{\uparrow \uparrow}^{N M}(t)$ also decrease with increasing $N$ if $N>10$.

\begin{tabular}{cccccccc}
\hline \hline $\mathrm{N}$ & $\mathrm{M}$ & $\max C_{\uparrow \uparrow}$ & $\max C_{\uparrow \downarrow}$ & $\max C_{\downarrow \downarrow}$ & $\max \tau_{\downarrow \downarrow}$ & $\max \Delta_{\uparrow}$ & $\max \Delta_{\downarrow}$ \\
\hline 4 & 2 & 0 & 0.412 & 0 & 1.000 & 0.889 & 0.889 \\
5 & 2 & 0.576 & 0.338 & 0.347 & 1.000 & 0.967 & 0.922 \\
6 & 2 & 0.372 & 0.295 & 0.225 & 0.919 & 0.877 & 0.789 \\
7 & 2 & 0.408 & 0.266 & 0.182 & 0.784 & 0.884 & 0.632 \\
8 & 2 & 0.364 & 0.244 & 0.141 & 0.610 & 0.849 & 0.495 \\
9 & 2 & 0.307 & 0.226 & 0.109 & 0.502 & 0.803 & 0.393 \\
10 & 2 & 0.253 & 0.211 & 0.084 & 0.401 & 0.755 & 0.311 \\
\hline & & & & & & & \\
6 & 3 & 0 & 0.275 & 0 & 1.000 & 0.960 & 0.960 \\
7 & 3 & 0.129 & 0.240 & 0.191 & 1.000 & 0.995 & 0.987 \\
8 & 3 & 0.111 & 0.216 & 0.115 & 0.973 & 0.950 & 0.928 \\
9 & 3 & 0.236 & 0.199 & 0.135 & 0.906 & 0.955 & 0.811 \\
10 & 3 & 0.205 & 0.174 & 0.092 & 0.643 & 0.886 & 0.560 \\
\hline & & & & & & & \\
8 & 4 & 0 & 0.209 & 0 & 1.000 & 0.980 & 0.980 \\
9 & 4 & 0.001 & 0.188 & 0.111 & 1.000 & 0.999 & 0.994 \\
10 & 4 & 0.000 & 0.173 & 0.034 & 0.993 & 0.983 & 0.967 \\
11 & 4 & 0.136 & 0.161 & 0.097 & 0.966 & 0.977 & 0.898 \\
\hline \hline
\end{tabular}

for the $\mathrm{W}$ state. However, the maximum of the other concurrence $C_{\downarrow \downarrow}^{41}(t)$ and tangle $\tau_{\uparrow \uparrow}^{41}(t)$ is already reached by the $\mathrm{W}$ state. Moreover, the residual tangles are zero during the whole evolution.

In general, for the equivalent-neighbor system with arbitrary number $N$ of QDs and only one of them having initially spin opposite to the others, we find that

$$
\Delta_{\uparrow}^{N 1}(t)=\Delta_{\downarrow}^{N 1}(t)=0,
$$

which implies that the genuine multipartite entanglement is not generated for $M=1$.

For systems with $M>1$, evolution becomes more complicated as seen in Figs. 4 and 5. In the present study, the most interesting for us are the maximum values of the concurrences and tangles as shown in table I for various numbers $N$ of QDs and their initial states as described by number $M$. For example, for any $N$ and $M=2$, we have

$$
\begin{aligned}
\left|\psi^{N 2}(t)\right\rangle= & \gamma_{0}^{N 2}(t)|\uparrow \uparrow \downarrow \downarrow\rangle+2 \gamma_{1}^{N 2}(t)|B B\rangle \\
& +\gamma_{2}^{N 2}(t)|\downarrow \downarrow \uparrow \uparrow\rangle,
\end{aligned}
$$

where $\gamma_{0,2}^{42}(t)=\left(e^{-i 4 \kappa t}+2 e^{i 2 \kappa t}\right) / 6 \pm 1 / 2$ and $\gamma_{1}^{42}(t)=$ $\left(e^{-i 4 \kappa t}-e^{i 2 \kappa t}\right) / 6$. Such states exhibit genuine multipartite entanglement as clearly shown in table I and Fig. 4. Let us analyze explicitly the simplest state among them, i.e., for $N=4$ and $M=2$. For evolution moment $\kappa t^{\prime}=\pi / 2$, the QD system evolves into the state

$$
\left|\psi^{N 2}\left(t^{\prime}\right)\right\rangle=\frac{1}{3}(|\uparrow \uparrow \downarrow \downarrow\rangle+2|B\rangle|B\rangle-2|\downarrow \downarrow \uparrow \uparrow\rangle),
$$

which does not exhibit pairwise entanglement $C_{\uparrow \uparrow}^{42}\left(t^{\prime}\right)=$ $C_{\uparrow \downarrow}^{42}\left(t^{\prime}\right)=C_{\downarrow \downarrow}^{42}\left(t^{\prime}\right)=0$ but exhibits the genuine multipar-

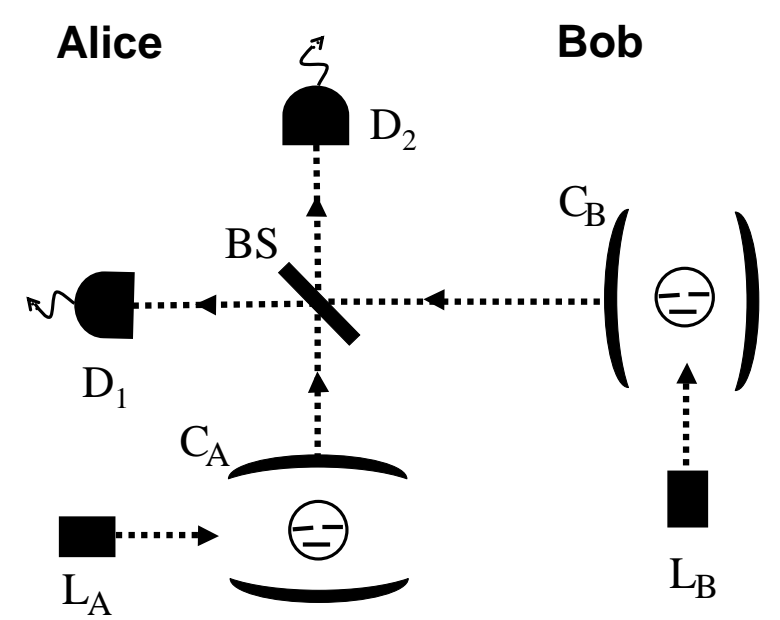

FIG. 6: Teleportation without insurance of electron-spin states via cavity decay based on the protocol of Bose, et al. [16]. Key: $C_{A}, C_{B}$ - microcavities, $D_{A}, D_{B}$ - photon counters, $L_{A}, L_{B}$ - lasers, BS - beam splitter.

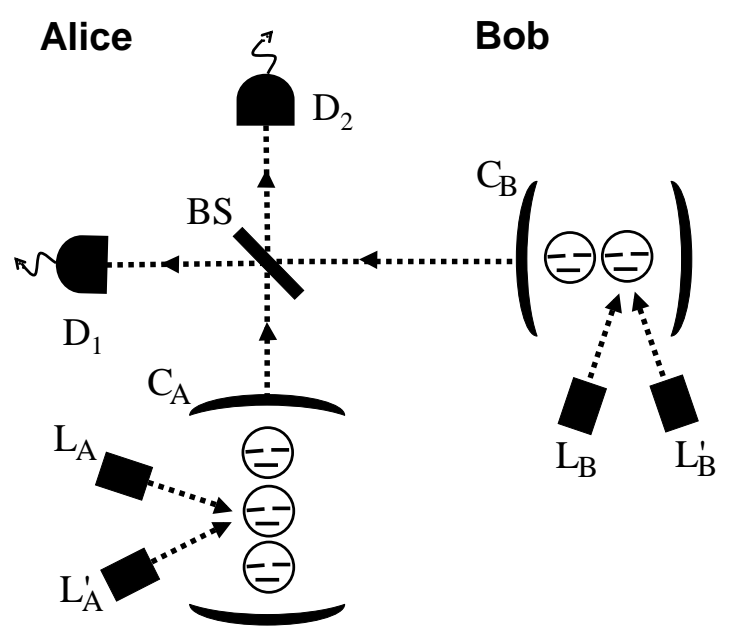

FIG. 7: Teleportation with insurance of entangled electronspin states via cavity decay in our generalized scheme.

tite entanglement, as described by the residual tangles $\Delta_{\uparrow}^{42}\left(t^{\prime}\right)=\Delta_{\downarrow}^{42}\left(t^{\prime}\right)=8 / 9$. This value is the highest during the whole evolution of $\left|\psi^{42}(t)\right\rangle$. Nevertheless higher degree of genuine multipartite entanglement can be observed by increasing number of QDs to $N=5$ for a fixed $M=2$ or by increasing both $N$ and $M$. A closer look at the data shown in table I, enables us to draw a conclusion that the highest degree of genuine multipartite entanglement, as quantified by the residual tangle, is generated in the discussed model for odd number $N=2 M+1$ of QDs and by initially preparing $M$ of them in the state with spin up (or spin down).

\section{TELEPORTATION OF ELECTRON-SPIN STATES}

Quantum teleportation of Bennett, et al. [29] is a method to transfer (information about) unknown quan- 
tum states over large distances via entangled particles and transmission of some classical information. Quantum teleportation is not just a curious effect but a fundamental protocol which enables universal quantum computation [30] as any quantum circuit can be realized using only quantum teleportation and single-qubit operations.

Here, we describe a protocol for quantum teleportation of electron-spin states of semiconductor QDs based on the protocol of Bose, et al. [16] (see also [17]) for teleportation of atomic states. The cavity spontaneous photon leakage plays a crucial role in this teleportation protocol. It is a well accepted fact that spontaneous decay of excited quantum systems is a mechanism of their decoherence and therefore usually plays a destructive role in QIP. However, Bose, et al. have shown how detection of decay can be used constructively not only for establishment of entanglement but also for the complete QIP such as teleportation. This surprising result can be understood by recalling the fact that a detected decay is a measurement on the state of the system from which the decay ensues.

Although, the original protocol of Bose, et al. was described for trapped atoms, it can, as well, be applied to teleportation of conduction-band electron-spin states of QDs with levels shown in Fig. 1. In the original scheme [16], it has been assumed that $\Delta \omega_{n}^{\uparrow}=\Delta \omega_{n}^{\downarrow}$ for $n=1,2$ but one can resign from this condition. An outline of the modified scheme is shown in Fig. 6. The setup consists of two optical cavities: Alice's $C_{A}$ and Bob's $C_{B}$ tuned to the same frequency $\omega_{\text {cav }}$. Each cavity contains a single three-level QD, which is illuminated within a proper period of time by classical laser field $\left(L_{A}\right.$ or $\left.L_{B}\right)$. By illuminating the QDs with the classical laser field of frequency $\omega_{n}^{L}$, Alice (designated by subscript $n=A$ ) and Bob $(n=B)$ can drive the transition between $|\downarrow\rangle_{n}$ and $|v\rangle_{n}$. The other transition between $|\uparrow\rangle_{n}$ and $|v\rangle_{n}$ is driven by the quantized cavity field of frequency $\omega_{\text {cav }}$. It is important to assume that detunings $\Delta \omega_{n}^{\uparrow}, \Delta \omega_{n}^{\downarrow}$ are large enough such that the lower levels $|v\rangle_{n}$ can effectively be decoupled (so neglected) from the evolution of the lower levels. Thus, we can assume that the quantum information is stored only in two levels $|\downarrow\rangle_{n}$ and $|\uparrow\rangle_{n}$. Both Alice's and Bob's cavities initially have no photons being described by vacuum state $|0\rangle_{n}$, and Bob's QD is initially in state $|\downarrow\rangle$. Alice does not know her QD state, which is of the form $|\psi\rangle_{A}=c|\downarrow\rangle_{A}+c^{\prime}|\uparrow\rangle_{A}$ (with the unknown coefficients $c$ and $c^{\prime}$ such that $\left.|c|^{2}+\left|c^{\prime}\right|^{2}=1\right)$. The main task is to teleport the state $|\psi\rangle_{A}$ to Bob. First, as a preparation of the state, Alice maps the $\mathrm{QD}$ state $|\psi\rangle_{A}$ on her cavity mode by illuminating her $\mathrm{QD}$ with the laser $L_{A}$ for a proper period of time. In the meantime, Bob illuminates his QD with the laser $L_{B}$ for another appropriate time period to generate a $\mathrm{QD}$-cavity-field entangled state $|\Psi\rangle_{B}=2^{-1 / 2}\left(|\downarrow\rangle_{B}|1\rangle_{B}+i|\uparrow\rangle_{B}|0\rangle_{B}\right)$, where $|1\rangle_{B}$ and $|0\rangle_{B}$ stand for the cavity state in vacuum or singlephoton state, respectively. Alice and Bob should synchronize their actions to finish simultaneously the preparations of their states since photons are leaking out from both of the cavities. Those photons are mixed on the 5050 beam splitter BS. Cavities are assumed to be singlesided so that the only leakage of photons occur through the sides of the cavities facing BS. The next step is the detection of the photons, when Alice just waits for a finite time period for click of the photon counter either $D_{1}$ or $D_{2}$. This detection of photons leaking from distinct cavities $C_{A}$ or $C_{B}$ constitutes a measurement that enables a transfer of quantum information from Alice's QD to Bob's QD. The cases, when Alice registers no clicks or two clicks, are rejected as the failure of the teleportation. At the post detection stage, Bob applies to the transferred state a proper phase shift depending on whether detector $D_{1}$ or $D_{2}$ clicked. This step corresponds to the correcting unitary transformation and completes the teleportation protocol. It is worth noting that the presented scheme, contrary to the original Bennett, et al. scheme [29], is probabilistic in the sense that the original state is destroyed even if the teleportation fails, which is the case when photon counters do not register one photon. Nevertheless, the scheme can be modified to a teleportation protocol with insurance by entangling the initial Alice's QD with a reserve QD also placed in her cavity $C_{A}$ to increase the probability of success. In Ref. [18], we have proposed a generalized scheme, depicted in Fig. 7 , that allows the teleportation of an entangled state of two atoms with insurance. Numerical calculations in Refs. $[18,19]$ showed that the average probability of success of our protocol is about 0.94 , while the average probability of successful teleportation without the insurance does not exceed 0.5. Our proposal for teleportation of QD spin states is a generalization of the scheme for teleportation of atomic-qubit states via cavity decay [16-19]. It is a multistage protocol and it is full description would exceed the recommended number of pages for the proceedings. Thus, the details will be presented elsewhere [31].

\section{DISCUSSION AND CONCLUSION}

Decoherence seriously limits the feasibility of the schemes especially by comparing decoherence rates in relation to the gate-operation times. But for simplicity in section III, we neglected decoherence effects in the analysis of quantum entanglement in the generalized models of Imamoğlu, et al. [9]. After Ref. [9], let us give a few estimations: The spin decoherence times of the conductionband electrons are relatively long and it is reasonable to assume to be $\sim 1 \mu s$. There are a few mechanisms of decoherence including spin-orbit coupling and cavity decay. The first decoherence mechanism is due to the coupling of the conduction-band electron spins to valence-band holes, which can result in decoherence of $\sim 1 n s$ and effective decoherence of $\sim 100 \mathrm{~ns}$. A more deteriorating effect is due to a short cavity lifetime $\Gamma_{\text {cav }}^{-1} \sim 10 \mathrm{ps}$, which can result in the effective decoherence of $\sim 1 n s$ [9]. By contrast, we discussed in section IV how to utilize this cavity decay in a constructive way for QIP. More details about decoherence of three-level systems in leaky cavities can be found in our papers [17-19]. Also our estimations of the sizedependent decoherence of large semiconductor QDs can be found [20]. Another serious obstacle to implement the discussed schemes is the requirement of strong coupling of a QD with a single photon. It seems that photoniccrystal microcavities could be a good solution, as they simultaneously exhibit a high quality factor (Q) in excess of 10,000 [32] and they are of an ultra-small, wavelengthscale modal volume. Although our results might appear highly theoretical, it should be noted that quantum en- 
tanglement of excitons in a single QD [33] and a QD molecule [34] has already been observed.

In this communication, we studied possibilities of generation of maximum pure-state bipartite and multipartite entanglement as well as mixed-state pairwise entanglement of electron spins in systems of QDs interacting via a microcavity field within the generalized models of Imamoğlu, et al. [9]. Conditions for generation of genuine multipartite entangled states of the conduction-band electron spins of QDs were discussed based on the CoffmanKundu-Wootters inequality. Such problems are important in the context of possible solid-state implementations of QIP including quantum teleportation. In particular, we briefly described a generalized protocol [31] of Bose, et al. [16] for teleportation (without and with insurance) of entangled spin-states of QDs between distant microcavities via their decay.

\section{Acknowledgments}

We acknowledge the support from the Japan Society for the Promotion of Science within the 21st Century COE Program and from the Polish KBN (grant No. 1 P03B $06428)$.
[1] S. L. Braunstein and H.-K. Lo (eds.), Scalable Quantum Computers: Paving the Way to Realization (Wiley-VCH, New York, 2001).

[2] D. D. Awschalom, D. Loss, and N. Samarth, Semiconductor Spintronics and Quantum Computing (Springer, New York, 2002).

[3] D. Loss and D. P. DiVincenzo, Phys. Rev. A 57, 120 (1998).

[4] J. A. Folk, et al., Science 299, 679 (2003); J. M. Elzerman, et al., Nature 430, 431 (2004); M. Kroutvar, et al., Nature 432, 81 (2004); M. Atatüre, et al., Science 312, $551(2006)$.

[5] T. Hayashi, et al., Phys. Rev. Lett. 91, 226804 (2003); J. R. Petta, et al., Science 309, 2180 (2005).

[6] M. N. Leuenberger, et al., Phys. Rev. Lett. 89, 207601 (2002).

[7] Y. Hirayama, et al., J. Phys.: Condens. Matter 18, S885 (2006).

[8] Ş. K. Özdemir, et al., e-J. Surf. Sci. Nanotech. 4, 669 (2006).

[9] A. Imamoğlu, et al., Phys. Rev. Lett. 83, 4204 (1999).

[10] J. P. Reithmaier et al., Nature 432, 197 (2004); T. Yoshie, et al., Nature 432, 200 (2004).

[11] A. Badolato, et al., Science 308, 1158 (2005).

[12] A. Miranowicz, et al., Phys. Rev. A 65, 062321 (2002).

[13] Yu-xi Liu, et al., Phys. Rev. A 65, 042326 (2002).

[14] Yu-xi Liu, et al., Phys. Rev. A 66, 062309 (2002).

[15] Yu-xi Liu, et al., J. Phys. A 37, 4423 (2004).
[16] S. Bose, et al., Phys. Rev. Lett. 83, 5158 (1999).

[17] G. Chimczak and R. Tanaś, J. Opt. B 4, 430 (2002).

[18] G. Chimczak, R. Tanaś, and A. Miranowicz, Phys. Rev. A 71, 032316 (2005);

[19] G. Chimczak, Phys. Rev. A 71, 052305 (2005).

[20] Yu-xi Liu, et al., Phys. Rev. A 67, 034303 (2003).

[21] C. C. Gerry and J. H. Eberly, Phys. Rev. A 42, 6805 (1990); M. Alexanian and M. Bose, ibid. 52, 2218 (1995); Y. Wu, ibid. 54, 1586 (1996).

[22] C. H. Bennett, et al., Phys. Rev. A 53, 2046 (1996).

[23] V. Coffman, J. Kundu, and W.K. Wootters, Phys. Rev. A 61, 052306 (2000).

[24] T. J. Osborne and F. Verstraete, Phys. Rev. Lett. 96, 220503 (2006).

[25] W. K. Wootters, Phys. Rev. Lett. 80, 2245 (1998).

[26] C. H. Bennett, et al., Phys. Rev. A 54, 3824 (1996).

[27] M. Koashi, V. Bužek, and N. Imoto, Phys. Rev. A 62, 050302(R) (2000).

[28] W. Dür, Phys. Rev. A 63, 020303 (2001).

[29] C. H. Bennett, et al., Phys. Rev. Lett. 70, 1895 (1993).

[30] D. Gottesman and I. L. Chuang, Nature 402, 390 (1999).

[31] G. Chimczak, et al., to be published.

[32] K. Srinivasan and O. Painter, Opt. Express 11, 579 (2003); H.-Y. Ryu, M. Notomi, and Y.-H. Lee, Appl. Phys. Lett. 83, 4294 (2003).

[33] G. Chen, et al., Science 289, 1906 (2000).

[34] M. Bayer, et al., Science 291, 451 (2001). 